\title{
Inflammatory myofibroblastic tumour in a 5-year-old child - a case report and review of the literature
}

\author{
Barbara Cuch ${ }^{1}$, Urszula Opasała ${ }^{1}$, Paweł Nachulewicz ${ }^{1}$, Justyna Szumiło ${ }^{2}$ \\ ${ }^{1}$ Department of Paediatric Surgery and Traumatology, Medical University of Lublin, Lublin, Poland \\ ${ }^{2}$ Department of Clinical Pathomorphology, Medical University of Lublin, Lublin, Poland
}

Videosurgery Miniinv 2014; 9 (4): 658-661

DOI: $10.5114 /$ wiitm.2014.45885

\begin{abstract}
Inflammatory myofibroblastic tumour is an uncommon tumour of intermediate malignant potential. Its aetiology is still unclear. It occurs predominantly in children and young adults. This report presents a case of pulmonary inflammatory myofibroblastic tumour in a 5-year-old girl. The patient had a history of recurrent respiratory tract infections. A chest radiograph and computed tomography chest scan showed a round mass in the lower lobe of her left lung. Thoracoscopic marginal excision of the tumour with an Endo-GIA stapler device (TYCO healthcare) was performed. Histological examination confirmed the final diagnosis of inflammatory myofibroblastic tumour. Postoperative recovery was uncomplicated and the patient was discharged 6 days after surgery. Round masses located in the lungs are very rare in children and the possibility of myofibroblastic tumour as well as metastatic lesions should be taken into consideration in such cases. Thoracoscopic excision is the best option in distally located lesions.
\end{abstract}

Key words: child, inflammatory myofibroblastic tumour, pseudotumour, paediatric surgery, pulmonary mass, lung.

\section{Introduction}

Inflammatory myofibroblastic tumour (IMT) is a rare entity which occurs predominantly in children and young adults. It is a tumour of intermediate malignant potential [1] and is usually located in the lungs, although other organs can also be affected. Diagnosis of IMT is still difficult. Its appearance and symptoms frequently mimic malignancy. Histological examination of surgically resected lesions is obviously required to make diagnosis. The pathogenesis of this condition is unclear; the possibilities include inflammatory reaction of the tissue and a true neoplastic process. There are many synonyms for IMT that reflect its diverse histological features, including plasma cell granuloma, fibrous histiocytoma, mast cell granuloma and xanthoma. Surgical resection remains the treatment of choice and to date has been reported to be highly effective [2].
This report presents a new case of pulmonary inflammatory myofibroblastic tumour diagnosed in a 5-year-old girl. The patient underwent thoracoscopy and radical mass excision.

\section{Case report}

A 5-year-old girl with a history of recurrent respiratory infections was admitted to hospital with productive cough, fatigue and fever. A chest radiograph revealed a round opacity in the left lung (Photo 1). Diagnostic assessment was extended to include chest computed tomography (CT) scan (Photos 2 $A-B)$. The round opacity in the superior segment of the left lower lobe was initially defined as a cystic space of bronchial origin. The patient was referred to the Department of Paediatric Surgery, Medical University of Lublin, Poland, and thoracoscopy was performed. Three $5 \mathrm{~mm}$ ports were inserted through

\section{Address for correspondence}

Barbara Cuch MD, Department of Paediatric Surgery and Traumatology, Medical University of Lublin, 6 Prof. Antoniego Gębali St,

20-093 Lublin, Poland, phone: +48 668029 310, e-mail: basia_cuch@o2.pl 
the intercostal space. A 30-degree camera was used to locate the lesion, which was accessible to marginal stapler resection. A $60 \mathrm{~mm}$ Endo stapler device (TYCO-healthcare) was introduced directly through the intercostal space. The mass was removed in a bag through the slightly enlarged port site and a drain was placed in the chest. Postoperative recovery was uneventful. The chest drain was removed on the second postoperative day and a control chest radiograph was performed. On the sixth postoperative day, the patient was discharged in good conditions and remains symptom-free 24 months after surgery. The removed tumour had a volume of $4.0 \mathrm{~cm} \times 3.0 \mathrm{~cm}$ $\times 3.0 \mathrm{~cm}$. It was whitish, soft and solid, and partly myxomatous on cross section.

Microscopic examination showed a hypercellular tumour composed mostly of interlacing bundles of spindle cells with collagenous stroma and smaller areas of myxoid stroma with stellate cells (Photos 3 A-B). Cellular and nuclear pleomorphism were not observed. Tumour cells were vimentin- and smooth muscle actin (SMA)-positive, but ALK1-, S-100 protein-, CD117-, bcl2- and pancytokeratin-negative. Dense infiltration of mixed inflammatory cells including T (CD3+) and B (CD20+) small lymphocytes, plasma cells and mast cells (CD117) was noted (Photo $3 \mathrm{~B}$ ). The proliferative index (Ki67) was low - about $5 \%$. Based on these data, a final diagnosis of inflammatory myofibroblastic tumour was established.

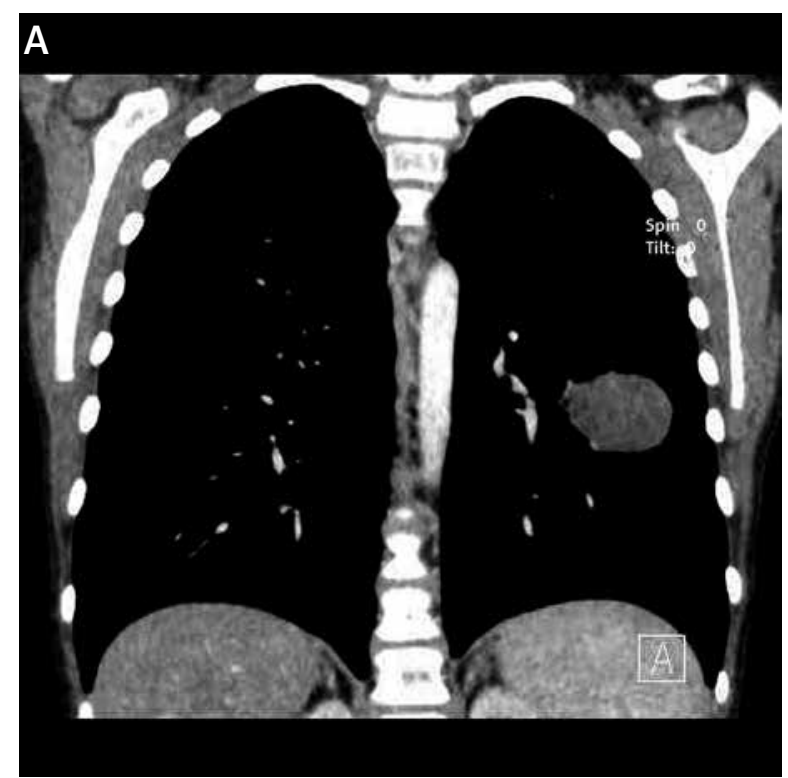

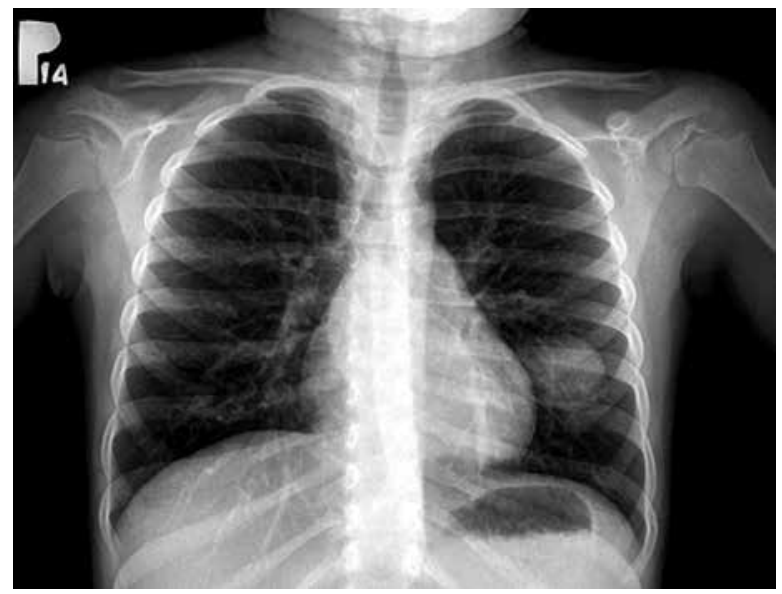

Photo 1. Chest radiograph showing a round opacity in the left lung

\section{Discussion}

The lung is the most common location for IMT, although occurrences in the urinary bladder, ovary, liver, kidney, gastrointestinal tract and soft tissues have also been reported. The IMT has the potential for local invasion and recurrences [2], so under the WHO histological classification it is an intermediate neoplasm. It was described for the first time by Brunn in 1939, but the name plasma cell granuloma was introduced in 1955 by Lane because many plasma cells were detected in the lesion. Primary pulmonary tumours are rare in children, and IMT accounts for $20 \%$ of them [3]. The aetiology of IMT is still unclear.

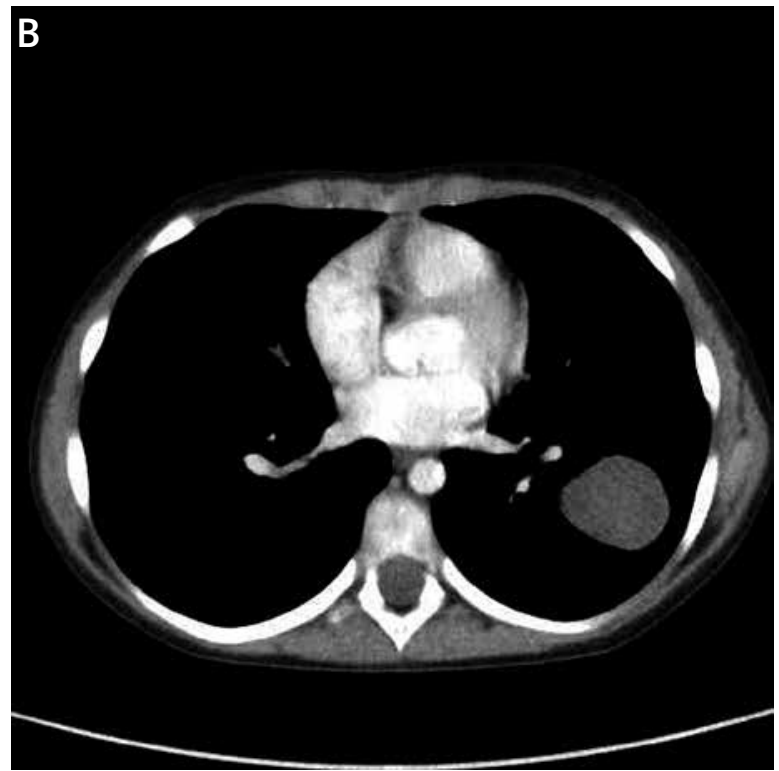

Photo 2. A - Chest computed tomography demonstrating a round mass in the superior segment of the left lower lobe. B - Chest computed tomography (axial section) demonstrating a round mass in the left lung 

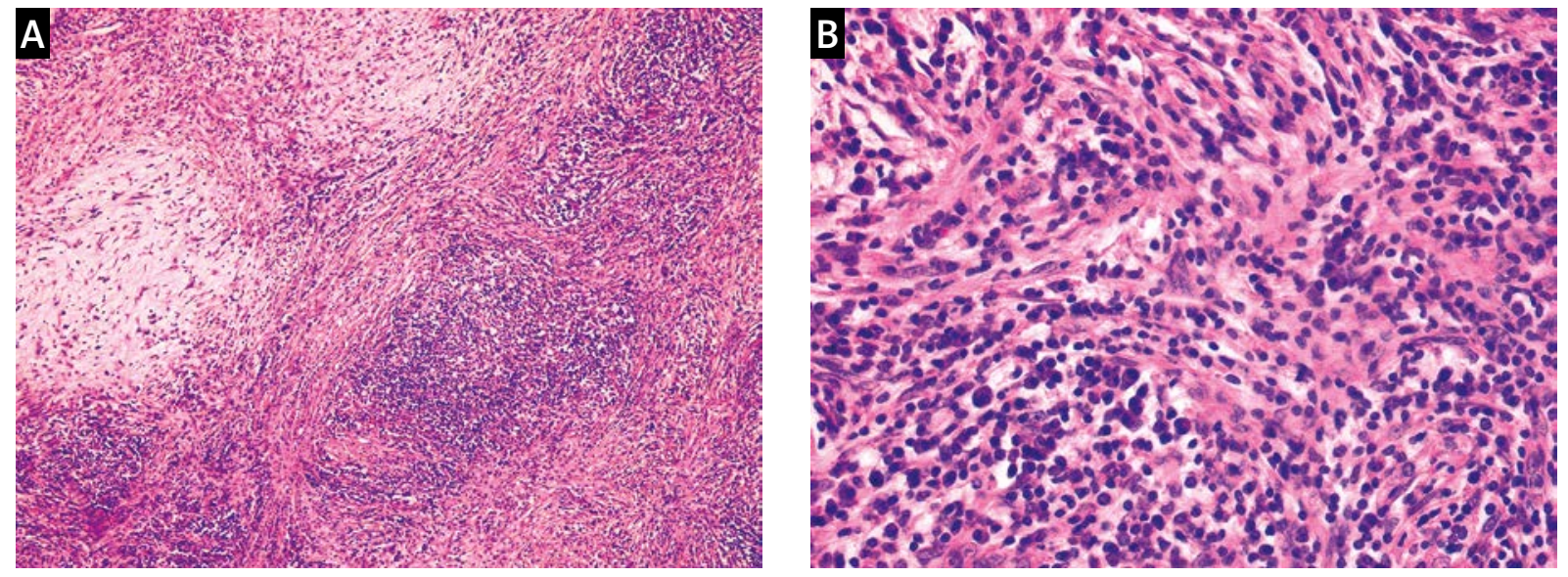

Photo 3. A - Inflammatory myofibroblastic tumour composed mostly of interlacing bundles of spindle cells with collagenous stroma and smaller areas of myxoid stroma with stellate cells (H+E; objective magn. $5 \times)$. B - Dense inflammatory infiltration composed of small lymphocytes, plasma cells and mast cells $(\mathrm{H}+\mathrm{E}$; objective magn. 20x)

Some studies have suggested that IMT is a true neoplasm on the basis of characteristic gene alterations such as fusion of the gene encoding anaplastic lymphoma kinase (ALK) on chromosome 2p23 and tropomyosin 3 (TPM3), which results in a functional chimeric protein [4]. This phenomenon (TPM3-ALK) is detected in $30 \%$ of paediatric IMT cases [5]. Other reports have suggested that the tumour is an inflammatory reactive lesion [6]. Antecedent respiratory infections have been reported in $1 / 3$ of patients and a wide variety of pathogens including Nocardia, Mycoplasma and Actinomyces have been reported to be involved. In some cases HHV-8 and EBV DNA sequences have been found in tumour cells. Coexistence of gene susceptibility and recurrent infection have also been reported [1]. Histopathologically, IMT has features characteristic of myofibroblastic proliferation (spindle cells) and a wide range of inflammatory cells, including lymphocytes, plasma cells, eosinophils and histiocytes [6].

The IMT typically appears as a solitary, slowly growing, well-demarcated and peripheral mass $1-10 \mathrm{~cm}$ in size [7]. Up to $70 \%$ of patients present some non-specific symptoms such as cough, fever, pain and haemoptysis; the clinical manifestations depend on location of the tumour [8]. Many patients are asymptomatic and the disease is discovered incidentally. It is difficult to establish a firm diagnosis before surgery; histological examination is always required. Only about $6 \%$ of patients are diagnosed on the basis of a biopsy specimen [9]. Bronchoscopic samples and fine needle aspiration biopsy are fre- quently insufficient as they may contain only inflammatory cells [10].

Surgical resection is the treatment of choice. Complete excision seems to be curative and prevents recurrences. Radiotherapy, chemotherapy or corticosteroid therapy can also be given where surgical intervention is contraindicated, and where there is multifocal disease or incomplete removal of the mass. Treatment with non-steroidal anti-inflammatory drugs (NSAIDs) has also been reported. These non-surgical treatments can achieve tumour shrinkage or even resolution. Operative technique depends on tumour location, the existence of local invasion and the patient's age and condition. Surgical management takes the form of mass excision, lobectomy or pneumonectomy, depending on tumour size and location. The most controversial aspect of surgery, especially in small children, is the resection of a mass arising from the main stem of the bronchus. Tumours in this location mostly require a total pneumonectomy; this usually has serious consequences for children. They may be afflicted with life-threatening right post-pneumonectomy syndrome. The reduction of pulmonary tissue is usually significant and after pneumonectomy patients may develop chest or spinal deformities and fail to thrive. Recent standards recognise the utility of a less destructive sleeve lobectomy in cases with nodal status limited to N2. The operative technique is more difficult than pneumonectomy, and should only be carried out by experienced surgeons, especially in small children and infants. According to the 
literature (less than 15 reported cases), the youngest child who has undergone sleeve lobectomy was 4 years old [11]. Advances in endoscopic technique have enabled thoracoscopic surgery to be performed successfully in children [12], but the predominant treatment in cases that have been reported is thoracotomy. For obvious reasons, wedge resection is an appropriate treatment only if complete resection is possible, although some authors have suggested that re-resection can be used to treat recurrences.

We performed thoracoscopic wedge mass resection from the superior segment of the left lower lobe. This management strategy seems to be curative, as the patient remains disease free. The survival rate following radical resection of IMT is excellent. Up to $90 \%$ of patients experience no recurrent tumours and remain symptom free [13]. Early diagnosis is crucial for the possibility of tissue-sparing treatment; most patients are diagnosed at an advanced stage. Spontaneous regression of IMT has been reported. The local invasiveness may result in aggravating health conditions and occasionally death. The recurrence rate after surgery ranges from 1.5 to $4 \%$. Long-term follow-up of patients after IMT resection is always required as recurrences have been reported 11 years after primary tumour resection [6]. Recurrence appears mostly as locally invasive tumours which exceed the lung (recurrence rate is about 46\%). Complete resection and tumour containment within single organs seem to be equally important factors [7]. The frequency of recurrent disease is increased in cases with incomplete resection, larger tumours, deep location, presence of the susceptibility gene (ALK), and in younger patients [8]. Five-year survival after resection exceeds 91\% [4].

\section{Conclusions}

Inflammatory myofibroblastic tumour occurs only rarely in children but should be considered in all cases of pulmonary lesions until it is excluded by histological examination. Complete excision is usually curative and adjuvant therapy is unnecessary.

\section{References}

1. Goel P, Bhatnagar V, Jain V, et al. Locally invasive pulmonary in flammatory myofibroblastic tumors in children. J Indian Assoc Pediatr Surg 2012; 17: 135-7.

2. Hammas N, Chbani L, Rami M, et al. A rare tumor of the lung: inflammatory myofibroblastic tumor. Diagn Pathol 2012; 7: 83.
3. Shochat SJ, Weldon CB. Tumors of the lung and chest wall. In: Pediatric surgery. Coran AG, Adzick NS, Krummel T, et al. (eds.). Elsevier, Philadelphia 2012; 1: 567.

4. Ochs K, Hoksch B, Frey U, et al. Inflammatory myofibroblastic tumour of the lung in a five-year-old girl. Interact Cardiovasc Thorac Surg 2010; 10: 805-7.

5. Mehta B, Mascarenhas L, Zhou S, et al. Inflammatory myofibroblastic tumors in childhood. Pediatr Hematol Oncol 2013; 30: 640-5

6. Sengupta S, Chatterjee U, Bandyopadhyay R, et al. Primary pulmonary neoplasms in children: a report of five cases. Indian J Med Paediatr Oncol 2011; 32: 223-6.

7. Lee HL, Kim L, Lee KH, et al. A case of pulmonary inflammatory pseudotumor: recurrence appearing as several consolidative lesions after complete resection. Korean J Intern Med 2005; 20: 168-72.

8. Rasalkar DD, Chu WC, To KF, et al. Radiological appearance of inflammatory myofibroblastic tumour. Pediatr Blood Cancer 2010; 54: 1029-31.

9. Chen CK, Jan CI, Tsai JS, et al. Inflammatory myofibroblastic tumor of the lung - a case report. J Cardiothorac Surg 2010; 5: 55.

10. Takeda SI, Onishi Y, Kawamura T, et al. Clinical spectrum of pulmonary inflammatory myofibroblastic tumor. Interact Cardiovasc Thorac Surg 2008; 7: 629-33.

11. Pan W, Du L, Wu Y, et al. Successful sleeve lobectomy of inflammatory myofibroblastic tumor in a 4-year-old child. Ann Thorac Cardiovasc Surg 2013. Electronic link: https://www.jstage.jst. go.jp/article/atcs/advpub/0/advpub_cr.12.02143/_article

12. Koga H, Suzuki K, Nishimura K, et al. Traction sutures allow endoscopic staples to be used safely during thoracoscopic pulmonary lobectomy in children weighing less than $15 \mathrm{~kg}$. J Laparoendosc Adv Surg Tech 2013; 23: 81-3.

13. Berman M, Georghiou GP, Schonfeld T, et al. Pulmonary inflammatory myofibroblastic tumor invading the left atrium. Ann Thorac Surg 2003; 76: 601-3.

Received: 16.04.2014, accepted: 1.09.2014. 